\title{
Quantum Adiabatic Approximation, Quantum Action, and Berry's Phase
}

\author{
Ali Mostafazadeh* \\ Theoretical Physics Institute, University of Alberta, \\ Edmonton, Alberta, Canada T6G 2J1. \\ June 1996
}

\begin{abstract}
An alternative interpretation of the quantum adiabatic approximation is presented. This interpretation is based on the ideas originally advocated by David Bohm in his quest for establishing a hidden variable alternative to quantum mechanics. It indicates that the validity of the quantum adiabatic approximation is a sufficient condition for the separability of the quantum action function in the time variable. The implications of this interpretation for Berry's adiabatic phase and its semi-classical limit are also discussed.
\end{abstract}

Probably one of the best recognized applications of the quantum adiabatic approximation [1, 2] is in Berry's derivation of the adiabatic geometrical phases [3]. Following Berry's, by now, classical article on the adiabatic geometric phase [3], Hannay proposed a classical analogue of Berry's phase [4] and Berry [5] and Anandan [6] explored the semiclassical limit of Berry's phase and the classical analogue of the general, non-adiabatic geometric phase [7], respectively. The purpose of this note is to provide an alternative interpretation of the quantum adiabatic approximation which yields a natural approach to study the semi-classical limit of this approximation and consequently Berry's phase.

*E-mail: alimos@phys.ualberta.ca 
Consider the parameter-dependent quantum Hamiltonian:

$$
\hat{H}[R]=H(\hat{p}, \hat{x} ; R):=\frac{1}{2} g^{a b}(R)\left[\hat{p}_{a}-A_{a}(\hat{x} ; R)\right]\left[\hat{p}_{b}-A_{b}(\hat{x} ; R)\right]+V(\hat{x} ; R),
$$

where $\hat{x}=\left(\hat{x}^{1}, \cdots, \hat{x}^{\mathcal{N}}\right)$ and $\hat{p}=\left(\hat{p}^{1}, \cdots, \hat{p}^{\mathcal{N}}\right)$ denote the position and momentum operators, $R=\left(R^{1}, \cdots, R^{m}\right)$ are the coordinates of a parameter space $M, g^{a b}(R)$ are the entries of a positive-definite symmetric invertible $R$-dependent matrix, and $A_{a}$ and $V$ are arbitrary vector and scalar potentials. Furthermore, let $\{|n ; R\rangle\}$ be a complete orthonormal set of eigenvectors of $\hat{H}[R]$ :

$$
\hat{H}[R]|n ; R\rangle=E_{n}[R]|n ; R\rangle .
$$

Then a curve $C:[0, T] \rightarrow M$ defines a time-dependent Hamiltonian according to: $\hat{H}(t):=$ $\hat{H}\left[R_{C}(t)\right]$, where $R_{C}(t)$ are coordinates of $C(t)$. In this case the energy eigenvectors and eigenvalues also become time-dependent: $|n ; t\rangle:=\left|n ; R_{C}(t)\right\rangle, E_{n}(t):=E_{n}\left[R_{C}(t)\right]$.

The dynamics of the corresponding quantum system is governed by the Schrödinger equation:

$$
i \hbar \frac{d}{d t}|\psi(t)\rangle=\hat{H}(t)|\psi(t)\rangle, \quad|\psi(0)\rangle=\left|\psi_{0}\right\rangle
$$

The quantum adiabatic approximation [1] states that if the Hamiltonian depends on time adiabatically [2], then an eigenstate of $\hat{H}(0)$ evolves into an eigenstate of $\hat{H}(t)$. In particular if $E_{n}(t)$ is non-degenerate for all $t \in[0, T]$, then $\left|\psi_{0}\right\rangle=|n ; t=0\rangle$ implies [3]:

$$
\begin{aligned}
|\psi(t)\rangle & \approx e^{i \alpha_{n}(t)}|n ; t\rangle, \quad \alpha_{n}(t)=\delta_{n}(t)+\gamma_{n}(t), \\
\delta_{n}(t) & :=-\frac{1}{\hbar} \int_{0}^{t} E_{n}\left(t^{\prime}\right) d t^{\prime}, \quad \gamma_{n}(t)=\int_{0}^{t} i\left\langle n ; t\left|\frac{d}{d t}\right| n ; t\right\rangle=\int_{R_{C}(0)}^{R_{C}(t)} A_{n}[R], \\
A_{n}[R] & :=i\langle n ; R|d| n ; R\rangle:=i\left\langle n ; R\left|\frac{\partial}{\partial R^{j}}\right| n ; R\right\rangle d R^{j} .
\end{aligned}
$$

Here the symbol $\approx$ is used to emphasize that the corresponding relation is an approximation. If the curve $C$ is closed, i.e., $C(T)=C(0)$ then $\hat{H}(t)$ is a periodic Hamiltonian. In this case, the phase angles $\alpha_{n}(T), \delta_{n}(T)$, and $\gamma_{n}(T)$, are called the adiabatic total, dynamical, and geometrical (Berry) phase angles.

In order to investigate the semi-classical (WKB) limit of the quantum adiabatic approximation, consider the position representation of the quantum system. In this case, the state vectors $|\psi\rangle$ are represented by wave functions $\langle x \mid \psi\rangle=\psi(x)$. In this article, I shall only consider the case where $\psi$ is a complex-valued square integrable function of $x \in \mathbb{R}^{\mathcal{N}}$. 
Following Bohm [8], one can write $\psi=\sqrt{\rho} \exp [i S / \hbar]$, where $\rho$ and $S$ are real-valued functions and $\rho$ is positive semi-definite. In terms of $\rho$ and $S$ the Schrödinger equation (3) is written in the form:

$$
\begin{aligned}
\partial_{t} S(x ; t)+H(x, \nabla S(x ; t) ; t)+Q(x ; t) & =0, \\
\partial_{t} \rho(x ; t)+\nabla \cdot J(x ; t) & =0,
\end{aligned}
$$

where $H(x, \nabla S(x ; t) ; t)$ is the classical Hamiltonian $H(x, p ; t)$ evaluated at $p=\nabla S(x ; t)$, $Q:=-\hbar^{2} g^{a b}\left(\partial_{a} \partial_{b} \sqrt{\rho}\right) /(2 \sqrt{\rho})$ is the quantum potential, and $J$ is the probability current density whose components are defined by $J^{a}:=\rho g^{a b}\left(\partial_{b} S-A_{b}\right)$. There are two well-known properties of Eqs. (7) and (8). Firstly, for $Q=0$, Eq. (đ) becomes identical with the Hamilton-Jacobi equation [9]. Therefore, in this case the phase angle $S=S(x ; t)$ of the wave function $\psi(x ; t)$ is nothing but the classical action function. This justifies the name "quantum action" for the general case where $Q$ does not vanish. In this general case, one can attempt to solve (7) and (8) by an iterative method in the first step of which one approximates $Q$ by zero. This approximation is know as the semi-classical or WKB approximation, [10, 11]. Secondly, Eq. (8) is a continuity equation corresponding to the conservation of the probabilities.

Similarly, one can express the eigenvalue equation (2) in the position representation. Using the notation

$$
\begin{gathered}
\langle x \mid n ; R\rangle=: \psi_{n}(x ; R)=: \sqrt{\rho_{n}(x ; R)} e^{i S_{n}(x ; R) / \hbar} \\
Q_{n}:=-\frac{\hbar^{2} g^{a b}\left(\partial_{a} \partial_{b} \sqrt{\rho_{n}}\right)}{2 \sqrt{\rho_{n}}}, \quad J_{n}^{a}:=\rho_{n} g^{a b}\left(\partial_{b} S_{n}-A_{b}\right),
\end{gathered}
$$

one has:

$$
\begin{aligned}
H\left(x, \nabla S_{n}(x ; R) ; R\right)+Q_{n}(x ; R) & =E_{n}[R], \\
\nabla \cdot J_{n}(x ; t) & =0 .
\end{aligned}
$$

Again in the semi-classical limit (also indicated by $\hbar \rightarrow 0$ in the loop expansion of the path integral kernel [12]) $Q_{n}$ is neglected [10, 11.

Next let us examine the statement of the quantum adiabatic approximation in the position representation. In view of (田), one has:

$$
\rho(x ; t) \approx \rho_{n}(x ; R(t)), \quad S(x ; t) \approx S_{n}(x ; R(t))+\hbar \alpha_{n}(t) .
$$

In order to express $\alpha_{n}$ in terms of $\rho_{n}$ and $S_{n}$, one may naively attempt to differentiate both sides of the second relation in (11) and use Eqs. (7)-(11) to simplify the result. This would 
lead to a differential equation for $\alpha_{n}$ which implies the incorrect result: $\alpha_{n}=\delta_{n}$. The problem with this derivation is that the relations in (11) are not strict equations. They are only valid under the conditions of the adiabatic approximation [2]. In other words, in general there are other additive terms on the right hand sides of these relations which in the adiabatic approximation may be neglected. The time derivatives of these additional terms however may not be negligible. Therefore one is allowed to use the relations in (11) as equalities as far as no time-differentiation is directly performed. For example, one has $\nabla S \approx \nabla S_{n}, Q \approx Q_{n}$ by virtue of which Eqs. (7) and (8) take the form:

$$
\partial_{t} S(x ; t) \approx-E_{n}(t), \quad \partial_{t} \rho(x ; t) \approx 0
$$

Here use is also made of Eqs. (9) and (10). These equations can be integrated to yield:

$$
S(x ; t) \approx S_{n}(x ; 0)+f(t), \quad \rho(x ; t) \approx \rho_{n}(x ; 0)
$$

where $f(t):=-\int_{0}^{t} E_{n}\left(t^{\prime}\right) d t^{\prime}$. Eqs. (13) indicate that for an adiabatic quantum system, the quantum action $S(x ; t)$ with $S(x ; 0)$ corresponding to an initial energy eigenvector, is separable in the time variable, whereas the quantum potential is time-independent.

Next consider the transition amplitude

$$
\langle n ; t \mid \psi(t)\rangle=: e^{i \alpha_{n}(t)}
$$

which may be viewed as the definition of $\alpha_{n}(t)$. Expressing (14) in the position representation and differentiating the result with respect to time one obtains:

$$
\frac{d}{d t} \alpha_{n}(t)=-i \int d x^{\mathcal{N}} e^{\frac{i}{\hbar}\left(S-S_{n}-\hbar \alpha_{n}\right)}\left[\frac{1}{2}\left(\sqrt{\frac{\rho}{\rho_{n}}} \partial_{t} \rho_{n}+\sqrt{\frac{\rho_{n}}{\rho}} \partial_{t} \rho\right)+\frac{i}{\hbar} \sqrt{\rho_{n} \rho}\left(\partial_{t} S-\partial_{t} S_{n}\right)\right] .
$$

One can now use (10), (12), (11) and $\int d x^{\mathcal{N}} \rho=\int d x^{\mathcal{N}} \rho_{n}=1$ to simplify the integrand in (15). This yields:

$$
\frac{d}{d t} \alpha_{n}(t)=-\frac{1}{\hbar} E_{n}(t)-\frac{1}{\hbar} \int d x^{\mathcal{N}} \rho_{n}(x ; R(t)) \partial_{t} S_{n}(x ; R(t))
$$

which up on integration reproduces Berry's result (雨) with:

$$
A_{n}[R]=-\frac{1}{\hbar} \int d x^{\mathcal{N}} \rho_{n}(x ; R) \frac{\partial}{\partial R^{j}} S_{n}(x ; R) d R^{j} .
$$

An obvious implication of this equation is that if the energy eigenfunctions are real, such as in the case of simple Harmonic oscillator, then the geometric phase is identically zero. 
In the semi-classical approximation, one has $Q(x ; t)=Q_{n}(x ; t)=0$ and the quantum action becomes identified with the classical action function. Therefore the requirement of the adiabaticity of the Hamiltonian leads to the separability of the classical action in the time variable. In particular, if the energy eigenvalues are time-independent, then $S(x ; t) \approx$ $S_{n}(x ; 0)-E_{n} t$ which is exactly valid when the (classical) Hamiltonian is time-independent. In this case the $S_{n}(x ; 0)$ is called Hamilton's characteristic function [9].

Another implication of the adiabatic approximation is that the validity of the semiclassical approximation can be directly checked by computing the initial quantum potential

$Q(x ; 0)$. If $Q(x ; 0)$ is negligibly small then by virtue of $\rho(x, t) \approx \rho(x ; 0)$ it remains small throughout an adiabatic evolution, and the semi-classical approximation is valid.

\section{References}

[1] A. Messiah, Quantum Mechanics, Vol. 2, North-Holland, Amsterdam (1962).

[2] A. Mostafazadeh, "The Quatum Adiabatic Approximation and the Geometric Phase," hep-th/9606053.

[3] M. V. Berry, Proc. Roy. Soc. London A392, 45 (1984).

[4] J. H. Hannay, J. Phys. A: Math. Gen. 18, 221 (1985).

[5] M. V. Berry, J. Phys. A: Math. Gen. 18, 15 (1985).

[6] J. Anandan, Phys. Lett. A129, 201 (1988).

[7] Y. Aharonov and J. Anandan, Phys. Rev. Lett. 58, 1593 (1987); J. Ananadan and Y. Aharonov, Phys. Rev. D38, 1863 (1988).

[8] D. Bohm, Phys. Rev. 85, 166 (1952); Phys. Rev. 85, 180 (1952).

[9] H. Goldstein, Classical Mechanics, Addison-Wesley, Reading Massachusetts (1980).

[10] D. Bohm, Quantum Theory, Prentice Hall, New York (1951).

[11] L. I. Schiff, Quantum Mechanics, McGraw-Hill, New York (1955).

[12] B. S. DeWitt, Supermanifolds, Cambridge University Press, Cambridge (1992). 